2004-03-01

\title{
Using Weblogs in Scholarship and Teaching
}

David Wiley

david.wiley@gmail.com

Trey Martindale

Follow this and additional works at: https://scholarsarchive.byu.edu/facpub

Part of the Educational Psychology Commons

\section{Original Publication Citation}

Martindale, T. \& Wiley, D. (25). Using Weblogs in Scholarship and Teaching. Tech Trends, 49(2), 55-61.

\section{BYU ScholarsArchive Citation}

Wiley, David and Martindale, Trey, "Using Weblogs in Scholarship and Teaching" (2004). Faculty Publications. 995.

https://scholarsarchive.byu.edu/facpub/995 


\title{
Using Weblogs in Scholarship and Teaching
}

\author{
By Trey Martindale and David A. Wiley
}

$\mathbf{T}$

he web has become an important resource for teaching and learning (National Center for Educational Statistics [NCES], 2003). One reason the web is such a vital resource is that it allows almost anyone to contribute to its "holdings." Tools like HTML editors and FTP clients have made web publishing available to many teachers and students. However, the challenge of learning to use these tools has been a barrier to web publishing for many. Weblogs reduce the technical barriers to effective web publishing significantly.

Defining a weblog (also known as a "blog") is somewhat of a challenge. The genre began to emerge around 1997, when a number of websites appeared that were akin to travelogues, pointing users to new and interesting locations on the Internet. At that time the Internet was still largely non-commercial, and could be characterized as an uncharted frontier. These new travelogue websites consisted of links to and commentary on websites their authors found interesting. Barger was probably the first to suggest the term "web log" (Barger, 1999). Barger offered this definition of the term:

A weblog (sometimes called a blog or a news page or a filter) is a webpage where a weblogger (sometimes called a blogger, or a pre-surfer) 'logs' all the other Web pages she finds interesting. The format is normally to add the newest entry at the top of the page, so that repeat visitors can catch up by simply reading down the page until they reach a link they saw on their last visit (Barger, 1999).

Dave Winer, probably the most recognized pioneer of the blogging movement, offers this alternative definition:

Weblogs are often-updated sites that point to articles elsewhere on the web, often with comments, and to on-site articles. A weblog is kind of a continual tour, with a human guide whom you get to know. There are many guides to choose from, each develops an audience, and there's also camaraderie and politics between the people who run weblogs - they point to each other in all kinds of structures, graphs, loops, etc. (Winer, 2002).

A weblog is therefore a website with a certain collection of distinctive features. In 2005 these features generally include:

- Automatic formatting of content in the form of "headlines," followed by "entries," or "stories"

- Time- and date-stamp of entries

- Archiving of past entries

- A search function to search through all entries

- A "blogroll" - a list of other blogs read by the author(s) of the current blog

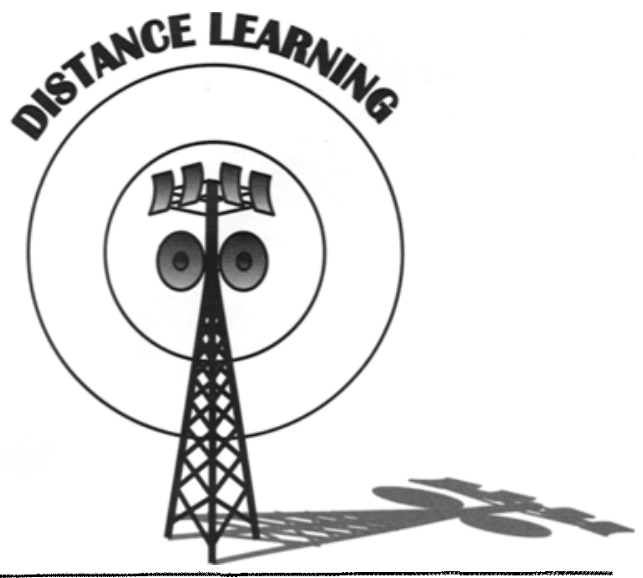


"From a visitor's point of view, a weblog is merely a website. But behind the scenes, the blog server software is actively creating a polished website that is archived, easily updated, searchable, interactive and potentially part of an extended topical discourse."
- A section associated with each entry where readers can post comments on the entry

-Simple syndication of the site content via RSS (Really Simple Syndication)

Typically, these features are all supported by blog server software, and the user (or "blogger") does not have to do any programming, or even formatting, to create a technically sophisticated, visually impressive website. (This says nothing about the site's content, of course). In the hands of more experienced web designers, blogs can usually be modified and customized in terms of appearance and functionality. Blogs can take on many different forms and appearances. To see examples of blog interfaces, and to find all the other links and supporting resources mentioned in this article, visit http://www.reusability.org/blogs/trey/archives/000601.html.

In the end, from a visitor's point of view, a weblog is merely a website. But behind the scenes, the blog server software is actively creating a polished website that is archived, easily updated, searchable, interactive and potentially part of an extended topical discourse. Adding entries to a blog (also known as "blogging") can be described as personal web publishing, or vanity press. For better or worse, blog server software bypasses the editorial function previously performed by print publishing companies, and therefore every blogger is a publisher.

From 2003 to 2005 the number of blogs on the web has increased at a very rapid rate, as commercial services like Blogger.com (http://www.blogger.com) have made creating a weblog both free and simple. As one indicator of the ubiquity of blogs, a search for "blog or weblog" via Google in November 2004 returned approximately $9,400,000$ results. A widely publicized survey by Perseus, a web data collection firm, (http://www.perseus.com/blogsurvey/thebloggingiceberg.html) found that many blogs are abandoned or very infrequently updated, while only a select few are regularly updated and widely read.

A key event in the brief history of blogs occurred during the war in Iraq in 2003. At the height of the initial conflict, a blog written by an Iraqi citizen (later dubbed the "Baghdad blogger" by the national news media) received tremendous publicity as the world sought war-related news. This unknown blogger published personal stories about the conditions in Baghdad during the bombing campaign, and solicited help in finding his missing friend. The personal and compelling nature of the account coupled with the timeliness of the content resulted in the blog being mentioned by the major U.S. television network news shows, which in turn introduced blogs for the first time to a large segment of the American public. In 2004 blogs were again prominently featured in the popular national press as a presidential candidate, Howard Dean, employed a blog to communicate with his supporters.

\section{Professional development through blogging and syndication}

A key feature of most blogs is the automated syndication of content - that is, blog content can be distributed easily and widely via a subscription model. The data format that enables this syndication is called RSS, which stands either for Really Simple Syndication, or Rich Site Summary, depending on which authority one consults. Most current blog server software supports this RSS subscription model.

Using an aggregator, one can "subscribe" to a particular blog (or many blogs), and have the content for each blog conveniently aggregated in one place (see Figure 1 , following page). An aggregator is a software application that collects RSS feeds and displays them, in a manner similar to the way that a Usenet news reader or an email client application aggregates and displays collections of messages. The key feature of aggregator software is the ability to collect (aggregate) RSS feeds from many different blogs and display the feeds in one convenient and coherent view. 


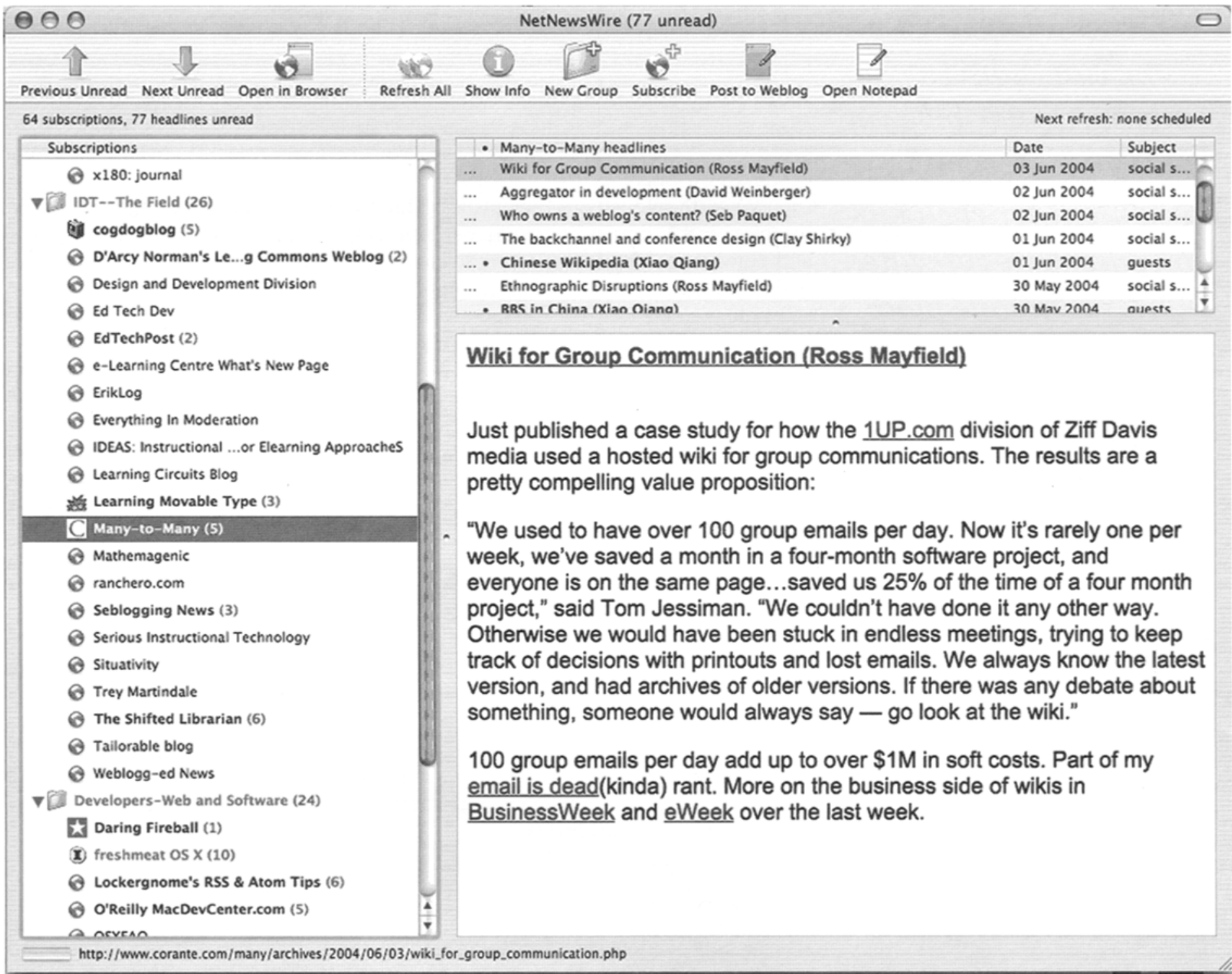

Figure 1. Netnews Wire aggregator interface.

Figure 1 is a screenshot of an aggregator called NetNewsWire, which has a three-paned interface. The left pane shows the titles of some of the blogs the user has subscribed to. The top pane shows recent entries from a selected blog. The center and largest pane shows the first few lines of the currently selected blog entry. Entries in normal weight text have already been viewed by the reader, while those in bold text are new and unread. There are a number of free and commercial aggregators for all major computer platforms.

\section{Using blogs in scholarship}

RSS aggregators are excellent tools for sifting through the deluge of information available from blogs related to instructional technology and other interesting topics. By using an aggregator a reader can quickly survey the blog landscape and receive notices of new content from a collection of blogs, as well as from a wide variety of sources like online newspapers and other periodicals. The content comes to the reader, rather than the reader having to seek the content from each individual site. For the authors as researchers, what once may have been a two hour daily ritual of surfing from site to site looking for new research articles 
"Using an aggregator, one can 'subscribe' to a particular blog (or many blogs), and have the content for each blog conveniently aggregated in one place." and other content has become a twenty-minute session of previewing and sifting through the information collected by the RSS aggregator.

Aggregators allow the individual reader either to sort through current RSSsyndicated content in significantly less time, or sift through a much greater amount of content within a given time period. As researchers and teachers who strive to stay on the leading edge of our field, we have found aggregators to be indispensable productivity tools.

We both publish our own professional blogs (Martindale at http://teachable. org/blog and Wiley at http://wiley.ed.usu.edu). These blogs have been a unique and convenient way for us to write about our own work and also direct others to interesting research, resources and viewpoints in instructional design and technology (IDT) as we interact with a network of colleagues, scholars and favorite writers across the Internet. We have found it professionally invigorating and challenging to read the writing of and interact with various thinkers within IDT and beyond. Regularly we discover individuals, groups and organizations who are contributing to our field via blogs and other syndicated content outlets. From this perspective blogging can be viewed as an extended, distributed conversation among participants. Having convenient access to syndicated professional content, opinion, research and discourse can be likened to being part of an engaged, extended, collegial faculty, but without the committee meetings.

While an extensive review of research on blogs is beyond the scope of this article, the reader may be interested in pursuing this topic. There is a small but growing body of research about the use of blogs in various environments, and also on the nature of communication within this "personal publishing" genre. "Into the Blogosphere" (http://blog.lib.umn.edu/blogosphere/) is a collection of essays on the nature of blog communication. Similarly the BROG Project (Blog Research on Genre, http://www.blogninja.com/), led by Susan Herring, contains a collection of blog-related research. Blogtalk (http://blogtalk.net/) is in its third year as a conference about blog research. And Lilia Efimova also maintains a significant collection of blog research (http://blog.mathemagenic.com/categories/ weblogResearch/index.html).

\section{Instructional Blogging}

In this section we describe how blogs and aggregators help students and instructors interact, build community and build a knowledge base in a formal instructional setting.

\section{Martindale's course experiences}

In a recent semester I went beyond my individual blog publishing and incorporated blogs into a graduate course I was teaching. In the fall of 2003 at East Carolina University we began a new doctoral program in educational leadership with a concentration in instructional development. That semester I taught a face-to-face seminar for eleven new doctoral students. I designed the seminar to examine the field of IDT, and give the students opportunities to explore topics germane to doctoral study like research design and dissertation preparation. As part of the course I provided a blog for each student, and gave the students some basic instructions about how to use a blog.

The major course assignments were to give a professional "conference-style" presentation and to write an article for a targeted scholarly journal. The blogs were key tools in improving the quality of student work on both these assignments. Students used the blogs to post their ideas on proposed article topics, and they posted links to relevant research and web resources along with their own commentary.

Each week of the course I required each student to choose a research study and give a 5-minute presentation and critique of the study. This activity served to 
immerse the students in important IDT literature since each week they would hear reports on eleven different IDT studies from scholarly sources. While this exercise was valuable, we faced an information management issue quickly, as students endeavored to organize this flood of research literature both physically and cognitively. The students possessed widely varying degrees of technical proficiency, yet all the students were able to publish their research summaries, critiques and citations to their own blogs easily. The blog structure enabled the creation of a distributed knowledge base of research literature consisting of approximately 99 research study critiques (11 students multiplied by 9 weeks). The critiques were stored by default in a searchable archive due to the features of the blog software.

For my students, the blogs offered a clear advantage over discussion forums because the blogs had greater sense of permanence. Discussion forums are usually inaccessible after a particular course has concluded, particularly if one is using a proprietary course management system like Blackboard or WebCT. I encouraged the students to continue blogging beyond the course, thereby building a collection of resources for current and future IDT students.

I also demonstrated aggregators and then required each student to use an aggregator and to subscribe to each other's blogs, so the students always knew when new resources or commentary were published by any member of the cohort. Clearly two keys to effective blogging are knowing who one's audience is, and knowing that an audience is in fact reading one's blog. My students were motivated and willing to write regularly and with clarity, knowing that fellow students and the instructor were reading the blogs.

Once the course ended, the student blogging also ceased. I had hoped that the blogs would continue, and in fact one or two of the students did create their own blogs, separate from the "institutionally-hosted" blog software. This raises the question of the institution hosting the student blogs. Initially I supported institutional hosting, but now I am against it. My experience is that a sense of ownership and full control over one's blog is important in supporting the blogging process. If the institution hosts the blog, what happens to the blog and its content when the student graduates? Who owns or controls the published content? Many companies now feature free blog services, so opportunities abound for creating individual blogs without institutional intervention.

In the future as I teach more courses (many of them completely online) I will need to decide whether I will have a blog for each course, or have one primary "teaching blog." Is each class a distinct audience, or is the content I publish of broad enough use and interest to each and every student who takes one of my courses? Or, do I continue to maintain one blog (as I do now) and not divide my limited readership into smaller audiences each of which is interested only in my "teaching," "research" or "service?" As one begins to think about and practice blogging, one soon realizes that the content and writing style must be for a particular audience - either actual or perceived. So students in a particular course may represent an audience, and simultaneously all students in our IDT program represent an audience. These various audiences will have some shared interests (for example the field of IDT) and some exclusive interests (course-specific material) when it comes to blog content. So in effect, I have multiple audiences, of varying sizes and levels of interest:

1) myself (with the blog serving as my personal web-based filing cabinet, reflective journal and "external brain")

2) students in my various courses

3) students in our degree programs

4) friends and co-workers at my institution

5) colleagues around the world with similar interests

6) unknown readers on the Web
"We consider blogs to be significantly easier to use than other web-related programs, including popular course management systems." 


\section{Wiley's course experiences}

In the fall of 2003 I (Wiley) team-taught with Janette Hill at the University of Georgia a survey course reviewing the literature in online learning research. Half the course participants were at UGA and half were at USU. Rather than trying to create accounts for students in one of the universities' formal learning management systems (e.g., WebCT), I created a course blog where we posted syllabi, assignments, news items and regular updates for students to access.

As we have described above, we consider blogs to be significantly easier to use than other web-related programs, including popular course management systems. Of course, blogs do not have some features, like live chat, that are available in CMS software, but we were willing to trade away some of these features for far greater ease of use in managing our course in a distributed fashion.

The blogroll on our course blog contained links to individual student blogs, where students were posting information relevant to team projects in which members were frequently located in different states. Because we were not posting intellectual property belonging to anyone other than ourselves, the publicly accessible blog worked as a very simple format for managing the day-to-day aspects of the course. Students in the course also participated in occasionally lively conversations using the asynchronous comment feature of the blogs. The instructors and the students subscribed to RSS feeds for the course and student blogs in order to keep up with newly posted entries and comments. As instructors, rather than surfing from site to site to see if and when students had posted, we simply used our aggregators to both track and read the student contributions.

Students appreciated being able to write without the extra step of needing to notify professors that writing assignments had been completed. Individuals and teams of students could subscribe to RSS feeds for team member blogs and be notified when teammates' websites were updated.

In the fall of 2004, I taught an online course about the culture of online interaction. Each week students were asked to spend five hours immersed in a different online communication medium (e.g., blogs, MOOs, IRC chat rooms, massively multiplayer games) and write mini-ethnographies about their experiences.

Having the students spend two weeks studying blogs and the culture of blogging paid great dividends. A few weeks into the semester, with students posting their weekly writing pieces to their blogs, we covered aggregators and I recommended that the students begin reading each other's blogs. A wonderful thing happened - students' weekly writings became longer and much more thoughtful. Students began commenting on each other's blogs (I had not required this purposefully, hoping that these interactions would emerge authentically). And additional pieces of writing (which I as instructor had not required) began appearing on their blogs.

Finally, when I began pointing to outstanding student posts on my own blog, some of my external readership began reading and commenting on the students' blogs. I received several enthusiastic emails and phone calls from students telling me how excited they were that "big names in the field" were reading their writing and engaging them in a dialog about issues related to online learning environments.

\section{Conclusion}

Blogs are currently receiving a great deal of attention in the popular media. In some cases blogs are being called "the next killer application," and "a replacement for email," among other lofty pronouncements. While a portion of this attention is warranted, in the end a blog is simply a website. But blogs may also represent an empowering technology - a step toward "leveling the (publishing) playing field." Blogs can certainly reduce the technical threshold for publishing on the web. 
Personal web publishing, as blogging is sometimes called, means that individual citizens can reach potentially a worldwide audience via the Internet.

Here is a brief example of a blog enabling one voice to be heard. I (Martindale) have a friend who is a pastor in a remote part of Central America in a place where many people live in poverty. The pastor has a number of supporters and friends in the region and around the world who have been deeply affected by the work he is doing to serve the poor and needy. One evening as we visited with the pastor, my wife explained to him what a blog was and how it functioned. Within the hour he had created his own blog at one of the few Internet cafés in his small city.

Now, without possessing any special technical skills or owning any expensive equipment, the pastor is able to publish news about his work to a very widely dispersed network of supporters around the world at a very low cost. The blog is hosted on a free service (supported by advertisements) so the pastor only has to pay for Internet access time. His "audience" may be less than fifty readers. But having a small readership is no longer a barrier to being published, at least online. In the world of print, content can only be published if it has financial backing, or potential for profit.

Furthermore, since the blog hosting service supports RSS, the pastor does not have to post on a regularly scheduled basis, nor do the pastor's supporters have to wonder when new content is published. They can subscribe to the blog's RSS feed and be notified when new content is posted.

We imagine that TechTrends readers have various "audiences" to address, and many individuals, groups and organizations with which they interact. Readers may also recognize the value of searchable, syndicated and archived communication among these sources. Blogs, as personal web publishing, represent one tool for enabling this kind of communication.

Dr. Trey Martindale is known for his work in the use of emerging technologies in the design of online learning environments. He is currently assistant professor at East Carolina University, where he teaches interesting and intelligent graduate students. Supporting information for this article and Dr. Martindale's other work can be found at http://teachable.org.

Dr. David Wiley is an assistant professor of Instructional Technology at Utah State University. He is an outspoken advocate for opening access to educational opportunity. Dr. Wiley's online home is http://wiley.ed.usu.edu.

\section{References}

Barger, J. (1999). Weblog resources FAQ. Retrieved March 18, 2004 from http://www.robotwisdom. com/weblogs/

National Center for Education Statistics (2003). Distance education at degree-granting postsecondary institutions: 2000-2001. NCES 2003-017. U.S. Department of Education: Washington, D.C.

Winer, D. (2002). The history of weblogs. Retrieved March 18, 2004 from http://newhome.weblogs. com/historyOfWeblogs
"A wonderful thing

happened - students'

weekly writings became

longer and much more

thoughtful. Students

began commenting on

each other's blogs ...

and additional

pieces of writing...

began appearing

on their blogs." 